\title{
Nina Tolksdorf
}

\section{Zu Kafkas Sprachen der Scham}

Abstract: Unterschiedlichsten Ansätzen und Theorien zufolge ist Scham zum einen subjektkonstitutiv und wird zum anderen mit Merkmalen wie dem Erröten und dem Versuch, sich zu verbergen, sich zu verstecken, versehen. Nicht so bei Kafka: Hier wird Scham vielmehr zu einem Erzählverfahren, das weiß, dass es sich nicht verstecken kann, weil seine Rhetorik immer für Sichtbarkeit sorgt. Der Artikel „Zu Kafkas Sprachen der Scham“ zeigt, dass Scham bei Kafka eine andere Richtung bekommt und wirft im Zuge dessen die Frage auf, ob Scham eine Möglichkeit aufweist, Verantwortung jenseits von Schuld und jenseits eines stabilen Subjekts zu denken.

Keywords: Scham; Franz Kafka; Rhetorik; Schuld; indirekte Rede; Verantwortung

Immer wieder werden Franz Kafkas Texte unter dem Vorzeichen der Schuld gelesen: Wessen hat sich Josef K. im Proceß schuldig gemacht? ${ }^{1}$ Was sind seine Verfehlungen? Warum ist die Schuld in der Strafkolonie „immer zweifellos“ (Kafka 1994, 212) und welche Rolle spielt sie im „Brief an den Vater“? Paradoxerweise scheint sich im Proce $\beta$ die Frage nach der Schuld besonders dringlich zu stellen, denn hier liegt sie nicht auf der Hand. Die Forschung sucht jedoch immer wieder nach Ursachen für K.s Schuld und findet sie in sexuellen Verfehlungen, in seiner Schamlosigkeit oder in seiner Schwäche und Uneinsichtigkeit. ${ }^{2}$ Wenn sie sich auf die Ursachen für K.s Schuld konzentriert, lässt sie jedoch eine bedeutendere Überlegung außer Acht: Sie bezweifelt nicht, dass es tatsächlich eine Verfehlung K.s gibt. Dass K. ermordet wird, geht hier mit der Sicherheit einher, dass dieser Mord von einer höheren (metaphysischen) Instanz gerechtfertigt ist, deren Autorität nicht in Frage gestellt wird. ${ }^{3}$ Mit anderen Worten: K. muss sterben, weil er schuldig ist, und er ist schuldig, weil er hingerichtet wird. Aber weder K. noch die Leserinnen und Leser erfahren, wie das Urteil des Gerichts ausgefallen ist falls es ein solches gegeben hat - oder in welcher Beziehung die Männer, die K.

1 Passagen dieses Beitrages sind auch im Kafka-Kapitel des Buches Performativität und Rhetorik der Redlichkeit. Nietzsche - Kleist - Kafka - Lasker-Schüler erschienen (Tolksdorf 2020).

2 Zur Frage der Schuld in der Proceß-Forschung vgl. Manfred Engel im Kafka-Handbuch (Engel und Auerochs 2010, 198).

3 Deleuze und Guattari zufolge ist die Frage nach Schuld oder Unschuld hingegen die Frage nach dem Unendlichen bzw. nach einer transzendentalen Institution und deswegen nicht Kafkas Frage (vgl. Deleuze und Guattari 1976, 73).

2 Open Access. () 2021 Nina Tolksdorf, published by De Gruyter. (c))BY-NC-ND This work is licensed under the Creative Commons Attribution-NonCommercial-NoDerivatives 4.0 International License. 
in den Steinbruch führen und töten, mit dem Gericht stehen und welcher Taten oder Untaten K. angeklagt wurde, falls diese Anklage überhaupt stattgefunden hat. Ein zweiter, nicht minder problematischer Aspekt der Schuldfrage ist, dass Schuld ein Individuum oder ein Subjekt impliziert, das sich entweder schuldig gemacht hat oder durch ein Verfahren im Namen der Schuld erst konstituiert wird. ${ }^{4}$ Das heißt, die Schuldfrage setzt ein Subjekt voraus, das in der Lage ist, seine Handlungen $\mathrm{zu}$ rechtfertigen oder die eigene Geschichte kohärent und in einem geschlossenen Narrativ wiederzugeben, wodurch die Subjektposition verteidigt oder geschaffen wird.

Dass aber zum Beispiel im Proceß gar kein stabiles Subjekt formuliert wird, das zu einem solchen Narrativ in der Lage wäre, zeigt sich an der Unmöglichkeit, die Frage „Wer spricht?“ zu beantworten. Vielmehr wird die Herkunft der Rede maximal verwirrt. Bereits der erste Satz zeugt davon: „Jemand mußte Josef $K$. verleumdet haben, denn ohne daß er etwas Böses getan hätte, wurde er eines Morgens verhaftet.“ (Kafka 1990, 9) Weil es hier nicht heißt, „Jemand hatte K. verleumdet“, sondern weil Kafka mit der Ambivalenz des „musste verleumdet haben“ arbeitet, wird, wie Beda Allemann zeigt, die Verleumdung als Sprechakt sowohl einer Notwendigkeit unterstellt als auch in Ungewissheit belassen (vgl. Allemann 1963, 239). Diese doppelte Valenz des „musste“ wird, wie Vogl zeigt, durch eine „Vermischung von direkter und indirekter Rede“ potenziert, durch die sich eine Stimme artikuliert, „in der ein Ich nicht aufhört, in der Maske des Er zu sprechen“ (Vogl 1994, 751). Die Antwort auf die Frage „Wer spricht?“ muss sich im Proceß insofern verlieren, als sie an einen Ursprung der Rede gerichtet ist, der sich eigentlich in einem Subjekt bündelt, hier aber nicht auffindbar ist. So schreiben Deleuze und Guattari, dass sich bei Kafka die Sprache „nicht zwischen etwas Geschehenem (oder Gefühltem) und etwas Gesagtem bildet“, sondern „immer von einem Sagen zum nächsten geht.“ (Deleuze und Guattari 1976, 107). Dadurch verweist das Ausgesagte ,weder auf ein Subjekt der Aussage als seine Ursache, noch auf ein Subjekt des Ausgesagten als seine Wirkung.“ (Deleuze und Guattari 1976, 26). Auch der erste Satz verweist nicht auf einen Ursprung/Urheber der Verleumdung, sondern auf ein „Jemand“. Die Fragen „Wer ist Jemand?“ und „Wer hat K. verleumdet?" markieren dann gleichsam die unlösbare Frage nach dem Ursprung der Sprache. Die Frage „Wer spricht?“, die eine Subjektposition sowie einen Ort des Subjekts forciert und immer von der Existenz eines Subjekts ausgeht (vgl. Derrida 1991), kann nicht beantwortet werden beziehungsweise wird die Antwort immer im gleichermaßen kollektiven und privaten „Jemand“ oder „Niemand“ verhallen.

4 Zur subjektkonstitutiven Kraft von Schuld vgl. vor allem die zweite Abhandlung von Friedrich Nietzsches Genealogie der Moral (Nietzsche 1999, ab 291). 
K. kommt damit die Funktion des man oder der vierten Person Singular zu, in der alles ,singulär und dadurch gleichzeitig kollektiv und privat, in eines besonders und allgemein, weder individuell noch universell“ (Deleuze 1993, 190) ist. Kurz: Die Suche nach einem schuldigen Subjekt muss am fehlenden Subjekt abgleiten.

Einige Vertreterinnen und Vertreter aus Psychologie, Soziologie, Philosophie und Anthropologie schreiben auch der Scham eine identitätsstiftende Funktion zu. Und obwohl Scham ein uferloses Thema ist, das sich nicht mit wenigen Worten zusammenfassen lässt, gibt es doch einige Merkmale, die Disziplinen übergreifend als konstitutiv für die Scham betrachtet werden. Ein Merkmal ist ihre Situierung an der Schwelle von Innen und Außen: Als Gefühl wird ihr ein Platz innerhalb des Körpers zugewiesen, gleichzeitig wird sie, z. B. im Erröten, oft sehr deutlich sichtbar. Der Begriff „Scham“ kommt von Verhüllen, Verbergen, Scham setzt aber ein Gesehen-Werden voraus. ${ }^{5}$ Ein zweites Merkmal ist ihre Situierung an der Schwelle von „Natur“ und „Kultur“. So meint zum Beispiel Freud, dass erst der aufrechte Gang des Menschen die Genitalien sichtbar und damit schutzbedürftig machte, so dass am Beginn des Kulturprozesses neben der Aufrichtung die Scham stehe (vgl. Freud 1994, 65). Sie befindet sich auch dann an dieser Schwelle, wenn sich für sogenannte natürliche Körperfunktionen und Begehren geschämt wird, weil sie in gewissen kulturellen Dynamiken als anstößig gelten und deswegen verdrängt, verboten oder verpönt werden. Weil die Scham ein „Affekt ist, der sich nicht gegen das richtet, was ihn erregt, sondern gegen denjenigen, der ihn hat" (Vogl 2010, 62-63) und sich das Ich so selbst erfährt, wird in der Folge auch der Scham eine Ich, Subjekt oder Identität erzeugende Wirkung zugeschrieben. So generiert nach Silvan Tomkins die Scham „the torment of self-consciousness“, das heißt in der Scham erfährt das Selbst sein Selbst (Tomkins 1995, 136) und auch laut Simmel ist die Scham die Betonung des Ich-Gefühls (vgl. Simmel 1983).

Schaut man sich die Scham bei Kafka genauer an, fallen vor allem zwei Verschiebungen auf: Erstens ist Scham bei Kafka nicht in erster Linie identitätsstiftend. Zweitens löst sie aus genau diesem Grund die nicht auffindbare Schuld ab und ist nicht als deren Manifestation zu verstehen. ${ }^{6}$ Die folgenden Lektüren einiger Figuren und Dynamiken in Kafkas Texten, die mit Scham verbunden sind, machen das deutlich.

Während gemeinhin Erröten, der Drang sich zu verstecken und Abwenden als körperlicher Ausdruck von Scham verstanden werden, sind es bei Kafka häufig

5 In diesem Punkt scheinen sich so unterschiedliche Denkerinnen und Denker wie Charles Darwin (1872 [1986] 333-337 und 317), Sigmund Freud (1994, 65), Georg Simmel (1983), Helmut Plessner (1981, 7-133), Silvan Tompkins (1995) und Eve Kosofsky Sedgwick (2003) einig zu sein. Wobei das „Wie“ des Gesehen-Werdens und das „Was“ sehr unterschiedlich ausfällt.

6 Vgl. dazu Vogl (2010, 62), anders z. B. Sokel $(1964,288)$ und Emrich $(1958,217)$. 
diejenigen Figuren, die aufrecht und erhoben sind, die deplatziert sind und sich preisgeben müssen, die mit Scham kurzgeschlossen werden. Die vielleicht eindrucksvollste Darstellung der Scham findet sich in einer sehr frühen Geschichte Kafkas, die er 1906 in einem Brief an Oskar Pollak schreibt:

\begin{abstract}
Der schamhafte Lange war in einem alten Dorf verkrochen zwischen niedrigen Häuschen und engen Gäßchen. So schmal waren die Gäßchen, dass, wenn zwei zusammen gingen, sie sich freundnachbarlich aneinander reiben mußten, und so niedrig waren die Stuben, dass, wenn der schamhafte Lange von seinem Hockstuhl sich aufreckte, er mit seinem großen eckigen Schädel geradewegs durch die Decke fuhr und ohne sonderliche Absicht auf die Strohdächer niederschauen mußte. ...

Vor Weihnachten einmal saß der Lange geduckt beim Fenster. In der Stube hatten seine Beine keinen Platz; so hatte er sie bequem aus dem Fenster gestreckt, dort baumelten sie vergnüglich. Mit seinen ungeschickten magern Spinnenfingern strickte er wollene Strümpfe für die Bauern. Die grauen Augen hatte er fast auf die Stricknadeln gespießt, denn es war schon dunkel. Jemand klopfte fein an die Plankentür. Das war der Unredliche in seinem Herzen. Der Lange riß das Maul auf. Der Gast lächelte. Und schon begann sich der Lange zu schämen. Seiner Länge schämte er sich und seiner wollenen Strümpfe und seiner Stube. Aber bei alldem wurde er nicht rot, sondern blieb zitronengelb wie zuvor. Und mit Schwierigkeit und Scham setzte er seine Knochenbeine in Gang und streckte schämig dem Gast die Hand entgegen. (Kafka 1983, 15)
\end{abstract}

Scham will sich bedecken, verbergen. „Scham versucht noch, verschämt, sich zu verhüllen“, sie entsteht aber nur, weil sie sichtbar, einem Blick ausgesetzt, Zeigen und Offenbarung ist (Lehmann 1991, 824); eine Bewegung, die sich in Kafkas Brief durch die dem Gast „schämig“ hingestreckte Hand, die zu allem Übel auch noch „durch die ganze Stube“ langt, manifestiert (Kafka 1983, 15). Auffällig ist, dass der Lange zitronengelb bleibt und nicht rot wird. Das weist einerseits darauf hin, dass er auch vor dem Besuch bereits schamhaft war, wie das sein Name verrät, andererseits wird Gelb dadurch zur Farbe der Scham. Die geläufige Redewendung ,vor Scham im Boden versinken zu wollen“ wird hier verkehrt, wenn der Schamhafte genau dies nicht tut, sondern im Gegenteil mit seinem eckigen Kopf durch die Decke fährt. Scham bekommt hier also buchstäblich eine andere Richtung. Deleuze und Guattari nannten die Geschichte vom schamhaften Langen eine „Meditation über Territorium und Haus“ (Deleuze und Guattari 1996, 219) und tatsächlich ist der größte Unterschied zwischen dem Langen und dem Unredlichen, dass der Erste allein wegen seiner Größe nicht in die „engen Gäßchen“ und „niedrigen Häuschen“ seines Dorfes passt, der Unredliche in die Stadt aber wie angegossen:

Der Unredliche in seinem Herzen, der wohnte in einer großen Stadt, die betrank sich Abend für Abend und war rasend Abend für Abend. Dieses ist nämlich der Städte Glück. Und wie die Stadt war so war auch der Unredliche in seinem Herzen. Dieses ist nämlich der Unredlichen Glück. (Kafka 1983, 15) 
Es ist genau dieses Unpassende, von dem aus sich Scham in Kafkas Texten ausbreitet und das auf moralischen, sexuellen, aber vor allem rhetorischen Ebenen ausgespielt wird. Das zeigt sich auch in der Erzählung ,Josefine, die Sängerin oder Das Volk der Mäuse.“ Das Pfeifen, so erfährt man, gehört zu den „gedankenlosen Gewohnheiten“ der Mäuse, das vor allem immer dann zu hören ist, wenn den Mäusen wohl ist (Kafka 1994, 354). Es sei deswegen anzunehmen, heißt es weiter, dass bei den Auftritten der Sängerin auch gepfiffen werde. Im Gegenteil sei es aber „mäuschenstill“ bei ihren Konzerten, bis einmal ,irgendein törichtes kleines Ding während Josefines Gesang in aller Unschuld auch zu pfeifen anfing. “(Kafka 1994, 354) Die anderen Zuhörerinnen ,zischten und pfiffen ... gleich die Störerin nieder, trotzdem es gar nicht nötig gewesen wäre, denn sie hätte sich gewiß auch sonst in Angst und Scham verkrochen“ (Kafka 1994, 355). In der unüblichen Situation der Mäuschenstille passiert das Übliche: Ein wohliges Pfeifen entgleitet der Maus und zieht den Ärger der anderen auf sich. Womit Kafka hier aber spielt, ist nicht nur das unpassende Pfeifen, das zur sozialen Ächtung führt, sondern vor allem auch die Redensart „sich vor Scham im Mauseloch verkriechen zu wollen“. In Anbetracht der Tatsache, dass hier Mäuse die Protagonistinnen sind, ist es nicht unwahrscheinlich, dass sie sich bereits in einem Mauseloch befinden. Selbst der Ort, der Schutz bieten soll, ist also vor der Scham nicht gefeit. Es gibt kein Entrinnen, vielmehr ist das Versteck der Ort der Scham. Der Begriff „mäuschenstill“ tut hier das Übrige: Er suggeriert, dass die absolute Stille eine typische Eigenschaft der Mäuse ist. Laut Erzählung trifft das jedoch nicht zu, sondern die Mäuschenstille bezeichnet gerade die unübliche Situation der Mäuse, zu deren ,gedankenlosen Gewohnheiten“ ja das Pfeifen gehört. Die Rhetorik des Textes stellt sich hier als unangemessen heraus, wie die pfeifende Maus beim Konzert Josefinens. Gerade dann, wenn sich die Rhetorik vor Angst und Scham verkriechen oder mäuschenstill bleiben will, indem sie sich in ihrem semantischen Umfeld tarnt, wird sie durch ihren unpassenden Ton laut und sichtbar. Vor der Scham gibt es kein Versteck, sie ist nur da, wenn und weil sie gesehen wird und sich nicht zurücknehmen lässt.

Dabei hat Scham keine eigene Sprache, sondern ist immer auch die Sprache der anderen: der Mäuse, des Jemand, des anderen Blicks. Das zeigt der schamhafte Lange, wenn er von den Worten des Unredlichen regelrecht befallen wird:

Der [Unredliche] drehte die Augenlider in die Höhe und die Worte gingen aus seinem Mund. Das waren feine Herren mit Lackschuhen und englischen Halsbinden und glänzenden Knöpfen, und wenn man sie heimlich fragte: ,Weißt du, was Blut aus Blut ist?‘ so antwortete einer anzüglich: ,Ja, ich habe englische Halsbinden.' Und kaum waren die Herrchen aus dem Munde draußen, stellten sie sich auf die Stiefelspitzen und waren groß, dann tänzelten sie zum Langen hin, kletterten zwickend und beißend an ihm hinauf und stopften sich ihm mühselig in die Ohren. (Kafka 1983, 15-16) 
Während der Unredliche die Herren aus seinem Mund gehen lässt, pikst er den Schamhaften mit einem Stock auf eine Weise, dass der Eindruck entsteht, er beschreibe ihn wie ein gelbes oder vergilbtes Papier. Die Zeichen, Buchstaben und Fragen, die an dem Schamhaften hochkriechen, wenn der Unredliche sich verabschiedet hat (vgl. Kafka 1989, 15), sind nicht die seinen, sondern diejenigen des Unredlichen.

Beide Aspekte, dass die eigene Sprache die der anderen ist und dass die Scham sich als unpassendes Moment manifestiert, prägen auch den Proceß. Das bezieht sich nicht nur auf die Fehlplatzierung K.s an den merkwürdigsten Orten, in versteckten Gängen, Hinterzimmern und fremden Betten, sondern vor allem auf die Eingabe, das heißt auf die Darstellung seines Lebens, die er kurz nach seiner Verhaftung anfängt zu schreiben. Die Eingabe und ihr Bezug zur Scham ist vor allem deswegen interessant, weil sich hier zeigt, dass die Sprache der Scham keine Identität erzwingen will, die ohnehin nicht erfüllt werden kann, weil sie, wie der Ursprung der Rede, nirgendwo zu finden ist.

Im ersten Kapitel des Roman-Projekts wird Joseph K. verhaftet. Weder er, noch die Leserinnen und Leser erfahren den Grund. Was durch die Verhaftung aber in Gang gesetzt wird, ist K.s Verteidigung, d.h. sein Versuch $\mathrm{zu}$ beweisen, dass er unschuldig ist. K. beginnt, von sich und seinem Leben Rechenschaft abzulegen. Da er nicht weiß, wofür er verhaftet wird, aber dennoch seine Unschuld beweisen will, nimmt er sich vor, eine Eingabe zu schreiben, in welcher er

eine kurze Lebensbeschreibung vorlegen und bei jedem irgendwie wichtigeren Ereignis erklären [wollte, N.T.], aus welchen Gründen er so gehandelt hatte, ob diese Handlungsweise nach seinem gegenwärtigen Urteil zu verwerfen oder zu billigen war und welche Gründe er für dieses oder jenes anführen konnte. (Kafka 1990, 118)

Dieses Vorhaben, die exakte und begründete Darstellung eines Lebens, erweist sich insofern als Gewaltakt, als es das Unmögliche von K. verlangt: „Man mußte keinen sehr ängstlichen Charakter haben und konnte doch leicht zu dem Glauben kommen, daß es unmöglich war, die Eingabe jemals fertig zu stellen.“ (Kafka 1990, 170)

Judith Butler hat anhand des Konzepts giving an account of oneself (vgl. Butler 2005) gezeigt, wie auch mit einem instabilen und dezentrierten Subjekt Verantwortung gedacht werden kann. Sie schreibt, dass das Konzept eines Subjektes, von dem nicht permanent gefordert wird, kongruent, transparent und sich selbst gleich zu sein und zu bleiben, einer ethischen Gewalt entgegenwirken könne, die eben darin bestehe, von sich und anderen zu fordern, immer mit sich selbst identisch zu sein (vgl. Butler 2001, 30-34). Das Subjekt kenne sich, so Butler, immer nur unvollständig und auch nur im Kontext eines sozialen Umfeldes, das ihm 
vorausgehe, das nach ihm existiere und es immer bereits in einer Form beeinflusst habe, die ihm nicht in Einzelheiten zugänglich werden könne:

The ,I‘ cannot tell the story of its own emergence, and the conditions of its own possibility, without in some sense bearing witness to a state of affairs to which one could not have been present, prior to one's own becoming, and so narrating that which one cannot know. (Butler 2001, 26)

Wie also, fragt Butler, können wir, wenn wir uns niemals vollständig gegenwärtig sind, Rechenschaft ablegen und einfordern, ohne permanent Gewalt ausgesetzt zu sein oder auszuüben? Wie kann Rechenschaft eingefordert werden, wenn Gründe und Ursachen des Handelns immer entgleiten? Sie diskutiert mit Bezug auf Adriana Cavarero (die sich wiederum auf Hannah Arendt bezieht) die Ethik der Frage „Wer bist du?“, 7 die wir nie aufhören dürfen zu stellen, weil sich das $\mathrm{Du}$ immer verändert, ebenso wie sich die Geschichten verändern, die das Du als Antwort gibt (vgl. Butler 2001 und 2005). Im Unterschied zur eingangs diskutierten Frage „Wer spricht?“ fragt Butler nicht nach der Instanz hinter und außerhalb der Sprache oder des Textes, sondern das Sprechen selbst rückt in den Fokus der Betrachtung. Die Frage nach dem Du darf Butler zufolge nicht nach Kongruenz und Geschlossenheit verlangen, sondern erwartet keine befriedigende Antwort und keinen abschließbaren Prozess. Deswegen muss die Frage auch immer wieder aufs Neue gestellt werden. Dem Gewaltakt des „Wer spricht?“ kann die Frage „Wer bist du? “, die sich an die Sprache richtet und nicht auf ein vermeintlich hinter der Sprache sich verbergendes Subjekt zielt, entgegengesetzt werden.

K.s „kurze Lebensbeschreibung“ soll nicht nur sein Leben transparent darstellen, sondern die Plausibilität seines Lebens beweisen, indem er für jede seiner Handlungen Gründe angeben und sie bewerten will. Motiviert wird die Eingabe K.s zwar durch den Vorwurf einer Schuld. Von Beginn an schreibt sich aber die Scham in sie ein:

Er erinnerte sich, wie er einmal an einem Vormittag, als er gerade mit Arbeit überhäuft war, plötzlich alles zur Seite geschoben und den Schreibblock vorgenommen hatte, um versuchsweise den Gedankengang einer derartigen Eingabe zu entwerfen und ihn vielleicht dem schwerfälligen Advokaten zur Verfügung zu stellen, und wie gerade in diesem Augenblick die Tür des Direktionszimmers sich öffnete und der Direktor-Stellvertreter mit großem Gelächter eintrat. Es war für K. damals sehr peinlich gewesen, obwohl der Direktor-Stellvertreter natürlich nicht über die Eingabe gelacht hatte, von der er nichts wußte, sondern über einen Börsenwitz, den er eben gehört hatte, einen Witz, der zum Verständnis eine Zeichnung erforderte, die nun der Direktor-Stellvertreter, über K.s Tisch gebeugt, mit K.s Blei-

7 Hannah Arendt untersucht in Vita Activa, inwieweit Handeln und Sprechen zeigen, wer jemand ist. (Vgl. Arendt 1960, 164) 
stift, den er ihm aus der Hand nahm, auf dem Schreibblock ausführte, der für die Eingabe bestimmt gewesen war. (Kafka 1990, 169)

Wenn der Direktor-Stellvertreter einen Witz auf das Papier zeichnet, das für die Eingabe bestimmt war, dann wird K.s Leben zum Witz. Auch hierin zeigt sich das Gewalttätige des Vorhabens, das aus dem Konzept der Schuld entsteht. Während die Schuld die kongruente Darstellung des Lebens verlangt, weiß die Scham, dass sie immer unpassend ist, dass also auch die Darstellung eines Lebens immer unpassend ist. Anders gesagt, Scham weiß, dass es keine bruchlose, alles erinnernde, stringente Darstellung gibt, bzw. weiß sie, dass das Vergessen und Brüche immer konstitutiver Teil der Darstellung sind. Deswegen ist es auch wieder die Scham, welche die Kulisse für K.s Tod bestimmt. Nachdem die Wächter im Kapitel „Ende“ K. abgeholt und in einen Steinbruch begleitet haben, heißt es:

Die Herren setzten K. auf die Erde nieder, lehnten ihn an den Stein und betteten seinen Kopf obenauf. Trotz aller Anstrengung, die sie sich gaben, und trotz allem Entgegenkommen, das ihnen K. bewies, blieb seine Haltung eine sehr gezwungene und unglaubwürdige. Der eine Herr bat daher den anderen, ihm für ein Weilchen das Hinlegen K.s allein zu überlassen, aber auch dadurch wurde es nicht besser. Schließlich ließen sie K. in einer Lage, die nicht einmal die beste von den bereits erreichten Lagen war. (Kafka 1990, 311)

Mit K.s Positionierung, die unelegant und unpassend ist, ist die Kulisse der Scham bereitet, die mit dem letzten Satz des Kapitels kulminiert:

Aber an K.s Gurgel legten sich die Hände des einen Herrn, während der andere das Messer ihm tief ins Herz stieß und zweimal dort drehte. Mit brechenden Augen sah noch K., wie die Herren, nahe vor seinem Gesicht, Wange an Wange aneinandergelehnt, die Entscheidung beobachteten. „Wie ein Hund!“ sagte er, es war, als sollte die Scham ihn überleben. (Kafka 1990, 241)

In der Forschung wird diese Scham oft zurückgeführt auf eine Schuld K.s bzw. wird sie als Zeichen dafür gelesen, dass sich K. schuldig gemacht hat und sich genau dafür schämt. ${ }^{8}$ Es heißt jedoch nicht, dass sich K. (für etwas) schämt, sondern dass es sei, „als sollte die Scham ihn überleben“. Dieser Unterschied ist

8 Sokel zufolge schämt sich K., weil er den Erwartungen der anderen Figuren nicht entsprechen konnte (vgl. Sokel 1964, 290), laut Emrich ist Scham bei Kafka immer Ausdruck eines sinnlosen Scheiterns (vgl. Emrich 1958, 217). Heidgen liest Scham als Resultat der Schuldigkeit des Subjekts (vgl. Heidgen 2013, 77). Anders sieht es z. B. Vogl, der meint, dass die Scham „Innervation einer Rede, aus der Ich und Nicht-Ich zugleich sprechen“, sei (Vogl 2010, 62). Andere Untersuchungen zur Scham bei Kafka liegen von Simons (2010), Danchev (2006), Ellrich (2004), Goltschnigg (1992), Kalka (2008) und Tiedemann (2005) vor. 
erstens deswegen wichtig, weil hier nicht mehr K., sondern die Scham Subjekt des Satzes ist und sie so weder einen Träger noch einen konkreten Inhalt hat oder ein (schuldiges) Subjekt konstituiert. Vielmehr wird sie zum Affekt, zu einem „free radical“, wie Sedgwick sagt, ,that attaches to and permanently intensifies or alters the meaning of - of almost anything“ (Sedgwick 2003, 62). ${ }^{9}$ Zweitens verzeichnet der Proce $\beta$ mit diesem Satz eine Verschiebung von Schuld zu Scham, ${ }^{10}$ in der zwar Verantwortung aufgefangen wird, weil sich K. seinem Urteil, wenn es denn ein solches gibt, nicht entzieht, die jedoch kein stabiles und kongruentes Subjekt voraussetzt. Während K. in der Schuldzuschreibung immer versuchte, seine Unschuld zu beweisen, übernimmt K. in der Scham die Verantwortung für das, was sich ihm entzieht; Verantwortung für etwas, das er weder kennt noch anerkennt. Als Affekt, der nicht versucht, Identität und ein Subjekt zu forcieren, überlebt die Scham am Ende des Proceß-Romans das Primat der Schuld. An einer früheren Stelle im Proceß heißt es „Es gab keine Schuld“ (Kafka 1990, 167). „Es gibt Scham“ scheint der letzte Satz zu sagen. Auch diese Scham will sich verbergen. Deswegen steht der Satz im Konjunktiv und unter dem Vorzeichen eines „es war, als sollte [...]“. Sie ist aber immer sichtbar und entsteht auch nur aufgrund dieser Sichtbarkeit. Auch der Konjunktiv kann sie nicht unerwähnt lassen, selbst wenn er sie als bloße Möglichkeit markiert. Es gibt aus der Scham kein Entkommen, ihre Rhetorik wird sie zeigen und für ihr Gesehen-Werden Sorge tragen. Ebenso gibt es aber in der Scham kein Entkommen aus der Verantwortung. Nur weil sich keine Schuld formuliert und sich kein Subjekt ausmachen lässt, bedeutet das nicht, dass sich keine Verantwortung finden lässt.

\footnotetext{
9 Anders geht Kalka davon aus, dass es immer einen Menschen geben muss, der Scham empfindet (vgl. Kalka 2008). Honold sieht in diesem letzten Satz eine Abspaltung der Scham von K. (Kafka 1995, 22), Simons verweist auf eine Verschiebung: „Das Subjekt verschwindet in der Scham." (Simons 2010, 289)

10 So auch Leon Wurmser, allerdings sieht auch er in der Scham eine Schuld. Laut Geisenhanslüke wird in der Scham ein Subjekt konstituiert (vgl. Geisenhanslüke 2006, 200). Schiffermüller hingegen schreibt: Die Scham „steht keineswegs im Zeichen der Schuld, wie oft behauptet wurde, sie bezeugt vielmehr, jenseits von gut und böse, ein ,letztes Lebensgefühl‘ ... Ähnlich wie die ,große Scham` des Zarathustra vor dem ,häßlichsten Menschen', die im Unterschied zu Kafka noch das antike eidos des Menschen hochhalten wollte, behauptet sie ein letztes ethos, das die Diskurse der Schuld und Rechtfertigung überlebt und Zeugnis ablegt für die Entstellung der menschlichen Gestalt“ (Schiffermüller 2011, 147).
} 


\section{Literaturverzeichnis}

Allemann, Beda. „Kafka: Der Prozess“. Der deutsche Roman vom Barock bis zur Gegenwart:

Struktur und Geschichte. Hg. Benno von Wiese. Düsseldorf: Bagel, 1963. 234-290.

Arendt, Hannah. Vita Activa - oder Vom tätigen Leben. Stuttgart: W. Kohlhammer, 1960.

Butler, Judith. „Giving an Account of Oneself.“ Diacritics 31.4 (2001): 22-40.

Butler, Judith. Kritik der ethischen Gewalt: Adorno-Vorlesungen 2002. Frankfurt/M.: Suhrkamp, 2003.

Butler, Judith. Giving an Account of Oneself. New York: Fordham University Press, 2005.

Danchev, Alex. „,Like a Dog!‘: Humiliation and Shame in the War on Terror.“ Alternatives: Global, Local, Political 31.3 (2006): 259-283.

Darwin, Charles. Der Ausdruck der Gemütsbewegungen bei dem Menschen und den Tieren. Stuttgart: E. Schweizerbart'sche Verlagshandlung, 1872.

Deleuze, Gilles. Logik des Sinns. Übers. Bernhard Dieckmann. Frankfurt/M.: Suhrkamp, 1993.

Deleuze, Gilles. Was ist Philosophie? Übers. Bernd Schwibs und Joseph Vogl. Frankfurt/M.: Suhrkamp, 1996.

Deleuze, Gilles, und Félix Guattari. Kafka: für eine kleine Literatur. Übers. Burkhart Kroeber. Frankfurt/M.: Suhrkamp, 1976.

Deleuze, Gilles, und Félix Guattari. Tausend Plateaus. Übers. Gabriele Ricke und Ronald Voullié. Berlin: Merve, 1997.

Derrida, Jacques. „,Eating well, ' or the Calculation of the Subject: An Interview with Jacques Derrida“. Who Comes After the Subject? Hg. Eduardo Cadava, Peter Connor und Jean-Luc Nancy. New York und London: Routledge, 1991. 96-119.

Ellrich, Lutz. „Diesseits der Scham: Notizen zu Spiel und Kampf bei Plessner und Kafka“. Textverkehr: Kafka und die Tradition. Hg. Claudia Liebrand und Franziska Schössler. Würzburg: Königshausen \& Neumann, 2004. 243-272.

Emrich, Wilhelm. Franz Kafka. Bonn: Athenäum, 1958.

Engel, Manfred, und Bernd Auerochs. Kafka-Handbuch: Leben - Werk - Wirkung. Stuttgart: J.B. Metzler, 2010.

Freud, Sigmund. Das Unbehagen in der Kultur: und andere kulturtheoretische Schriften. Frankfurt/M.: Suhrkamp, 1994.

Friedländer, Saul. Franz Kafka: The Poet of Shame and Guilt. New Haven und London: Yale University Press, 2016.

Geisenhanslüke, Achim. Masken des Selbst: Aufrichtigkeit und Verstellung in der europäischen Literatur. Darmstadt: Wissenschaftliche Buchgesellschaft, 2006.

Goltschnigg, Dietmar. „Die unendliche Scham: Das elende Ende Josef K.s im Kontext von Kafkas Leben und Werk". Literature, Culture and Ethnicity: Studies on Medieval, Renaissance and Modern Literatures. Hg. Mirko Jurak. London: Cambridge University Press, 1992. 181-191.

Heidgen, Michael. Inszenierungen eines Affekts: Scham und ihre Konstruktion in der Literatur der Moderne. Göttingen: V\&R Unipress, 2013.

Hiebel, Hans Helmut. Die Zeichen des Gesetzes: Recht und Macht bei Franz Kafka. München: Fink, 1989.

Honold, Alexander. „Exotische Verhandlungen: Fremdkörper in Kafkas ,Process““. Kafka verschrieben. Hg. Irmgard M. Wirtz. Göttingen: Wallstein, 2010.13-36.

Kafka, Franz. Briefe 1902-1924. Gesammelte Werke. Hg. Max Brod. Frankfurt/M.: Fischer, 1983.

Kafka, Franz. Der Proceß. Kritische Ausgabe. Hg. Malcolm Parsley. Frankfurt/M.: Fischer, 1990. 
Kafka, Franz. „Josefine, die Sängerin oder das Volk der Mäuse“. In: Drucke zu Lebzeiten. Hg. Wolf Kittler, Hans-Gerd Koch und Gerhard Neumann. Frankfurt/M.: Fischer, 1994. 350-380.

Kalka, Joachim. „Kafkas Sätze (51): Scham bedeutet Hoffnung“. Frankfurter Allgemeine Zeitung, 3. September 2008.

Lehmann, Hans-Thies. „Das Welttheater der Scham. Dreißig Annäherungen an den Entzug der Darstellung“. Merkur 45 (1991): 824-839.

Menninghaus, Winfried. Ekel: Theorie und Geschichte einer starken Empfindung. Frankfurt/M.: Suhrkamp, 1999.

Nietzsche, Friedrich Wilhelm. Sämtliche Werke. Kritische Studienausgabe: Jenseits von Gut und Böse, Zur Genealogie der Moral. Hg. Giorgio Colli und Mazzino Montinari, Bd. 5. Berlin und New York: De Gruyter, 1999.

Plessner, Helmut. „Grenzen der Gemeinschaft“. Gesammelte Schriften, Bd. 5. Frankfurt/M.: Suhrkamp, 1981. 7-133.

Schiffermüller, Isolde. Franz Kafkas Gesten: Studien zur Entstellung der menschlichen Sprache. Tübingen: Francke, 2011.

Sedgwick, Eve Kosofsky, und Adam Frank. Shame and Its Sisters: A Silvan Tomkins Reader. Durham: Duke University Press, 1995.

Sedgwick, Eve Kosofsky. Touching Feeling: Affect, Pedagogy, Performativity. Durham: Duke University Press, 2003.

Simmel, Georg. „Zur Psychologie der Scham“. Schriften zur Soziologie. Hg. Otthein Rammstedt und Heinz-Jürgen Dahme. Frankfurt/M.: Suhrkamp, 1983.140-151.

Simons, Oliver. „Schuld und Scham: Kafkas episches Theater“. Kafkas Institutionen. Hg. Arne Höcker und Oliver Simons. Wien: Transkript, 2007. 269-293.

Sokel, Walter. Franz Kafka - Tragik und Ironie; zur Struktur seiner Kunst. München: A. Langen, 1964. Tiedemann, Rolf, und Peter Krapp. „Kafka Studies, the Culture Industry, and the Concept of Shame: Improper Remarks between Moral Philosophy and Philosophy of History“. Cultural Critique 60.1 (2005): 245-258.

Tolksdorf, Nina. Performativität und Rhetorik der Redlichkeit. Nietzsche - Kleist - Kafka Lasker-Schüler. Berlin und Boston: De Gruyter, 2020.

Vogl, Joseph. „Vierte Person: Kafkas Erzählstimme“. Deutsche Vierteljahrsschrift für Literaturwissenschaft und Geistesgeschichte: DVJS 68.4 (1994): 745-756.

Vogl, Joseph. Ort der Gewalt. Kafkas literarische Ethik. Zürich: Diaphanes, 2010.

Wurmser, Leon. Die Maske der Scham. Berlin: Springer, 2012.

Nina Tolksdorf ist Postdoktorandin am Exzellenzcluster Temporal Communities: Doing Literature in a Global Perspective an der Freien Universität Berlin. Sie studierte Literatur, Philosophie und Komparatistik an der TU Berlin und in Aberdeen. In ihrer Dissertation, die sie 2017 an der Johns Hopkins University in Baltimore verteidigt hat, entwickelt sie mit Nietzsche, Kleist und Kafka einen performativen und rhetorischen Begriff von Redlichkeit. Ihr neues Projekt mit dem Arbeitstitel „Pantomime in Literatur, Film und Theater um 1900“ befragt anhand pantomimischer Darstellungen die Darstellungsverfahren der unterschiedlichen Medien. 
